\title{
SUR UNE FORME PARTICULIÈRE DE L'ÉQUATION D'HUGONIOT
}

PAR J.-R. FELLOUS *

\section{Introduction}

L'objet de cette note s'applique à l'écoulement tourbillon en fluide compressible dont l'étude conduit à une forme particulière de l'équation d'Hugoniot, équation classique bien connue qui relie la variation de section offerte à l'écoulement à la variation du nombre de Mach.

L'écoulement tourbillon plan en fluide parfait obéit à un certain nombre d'équations simples qui expriment d'une part les lois de la mécanique et traduisent d'autre part la géométrie de cet écoulement qui, en raison de sa symétrie de révolution, s'étudiera de préférence en coordonnées polaires.

1/

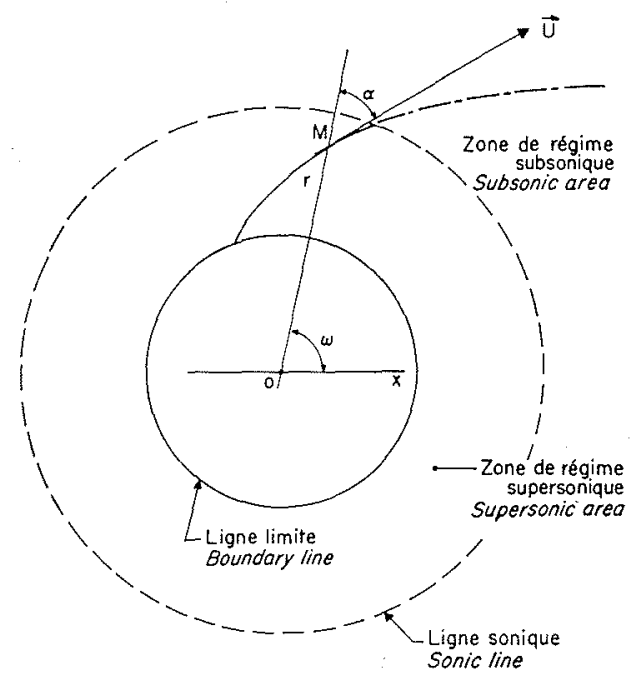

* Docteur ès Sciences, Ingénieur à SOGREAH.
Notations.

Le point courant sera repéré au moyen de ses rayon et angle polaires $r$ et $\omega$;

$\alpha$ : angle du vecteur vitesse avec le rayon polaire; on notera que la ligne isobare étant le cercle centré à l'origine et de rayon $r, \alpha$ désigne également l'angle du vecteur vitesse avec la normale à l'isobare;

$p, \rho, u, \mathrm{M}$ : pression statique, masse spécifique, vitesse et nombre de Mach de l'écoulement au point courant;

$M^{*}$ : nombre de Mach critique (rapporté à la célérité critique du son).

Mise

en équations de l'écoulement tourbillon

- L'équation $d p / d n=\rho u^{2} / \mathrm{R}$ exprime l'équilibre des forces centrifuges et des forces de pression statique normales à la ligne de courant.

-- Le vecteur gradient de pression statique est orienté suivant le rayon polaire, ce qui conduit à :

$$
\frac{d p}{d n}=\frac{d p}{d r} \sin \alpha
$$

- L'équation de quantité de mouvement donne:

$$
\frac{d p}{\rho}+u d u=0
$$




\section{J.-R. FELLOUS}

De ces deux premières équations, on déduit :

$$
\mathrm{R}=\frac{-u}{d u} \frac{d r}{\sin \alpha}
$$

- La continuité du débit masse s'écrit :

$$
2 \pi r \rho u \cos \alpha=\text { Cte }
$$

La section offerte à l'écoulement a donc la valeur $2 \pi r \cos \alpha$ et la relation d'Hugoniot classique prend done ici la forme:

$$
\frac{d(r \cos \alpha)}{r \cos \alpha}+\frac{d u}{u}\left(1-\mathrm{M}^{2}\right)=0
$$

L'élimination de $d u / u$ permet d'écrire :

$$
\mathrm{R}=\left(1-\mathrm{M}^{2}\right) \cdot \frac{r \cos \alpha}{d(r \cos \alpha)} \frac{d r}{\sin \alpha}
$$

Par ailleurs, $d \theta$ désignant la variation de l'angle repérant la direction du vecteur vitesse, c'est-à-dire la direction de la tangente le long de l'élément d'arc $d s$ suivant la trajectoire tourbillon, on a :

$$
d s=\operatorname{R} d \theta
$$

cet élément d'arc s'écrit, en coordonnées polaires :

et comme :

$$
d s^{2}=d r^{2}+r^{2} d \omega^{2}
$$

$$
d \theta=d \omega+d \alpha
$$

il vient finalement, en tenant compte de :

$$
\begin{aligned}
& \operatorname{tg} \alpha=r d \omega / d r \\
& \mathrm{R}=\frac{r d r}{d(r \sin \alpha)}
\end{aligned}
$$

L'élimination de $\mathrm{R}$ entre les équations (1) et (2) donne :

$$
\left(1-\mathrm{M}^{2}\right) \frac{d(r \sin \alpha)}{\sin \alpha}=\frac{d(r \cos \alpha)}{\cos \alpha}
$$

- Enfin, si on tient compte de l'invariance du moment cinétique spécifique $\sigma=r u \sin \alpha=$ Cte puisqu'on est en fluide parfait, et à nouveau de l'équation de continuité pru $\cos \alpha=$ Cte, ces deux dernières équations montrent en particulier que la masse spécifique est directement proportionnelle à $\operatorname{tg} \alpha\left({ }^{\star}\right)$, on aboutit à une forme particulièrement remarquable de l'équation d'Hugoniot appliquée à l'écoulement tourbillon :

$$
\frac{d r}{r}+\frac{d u}{u}\left(1-\mathrm{M}^{2} \cos ^{2} \alpha\right)=0
$$

Aux lieux et places de la section et du nombre de Mach dans l'équation d'Hugoniot classique, s'introduisent respectivement le rayon polaire et le

(**) On notera qu'en tluide incompressible $o=$ Cte : la trajectoire tourbillon est donc une spirale logarithmique. nombre $\mathrm{M} \cos \alpha$ qui est le produit du nombre de Mach par le cosinus de l'angle du vecteur vitesse avec la normale à la ligne isobare.

\section{Signification physique du nombre}

$M \cos \alpha$

L'équation :

$$
\frac{d r}{r}+\frac{d u}{u}\left(1-\mathrm{M}^{2} \cos ^{2} \alpha\right)=0
$$

gouverne l'écoulement tourbillon. Elle met en évidence :

1. l'existence d'un rayon limite qui est atteint quand $\mathrm{M} \cos \alpha=1$; l'écoulement puits-tourbillon ne peut se prolonger au-delà de ce rayon limite;

$2^{\circ}$ l'écoulement n'est pas commandé par la valeur du nombre de Mach mais par la valeur du nombre $\mathrm{M} \cos \alpha$; en particulier, un écoulement source-tourbillon supersonique peut revenir par voie purement isentropique en régime subsonique, à la condition que $\mathrm{M} \cos \alpha<1$.

Dans le cas particulier de l'écoulement tourbillon, cette remarque peut paraître évidente a priori. En effet, la géométrie de révolution de l'écoulement impose au front de l'onde de choc, si choc il y a, de se réduire à une surface cylindrique de révolution d'axe confondu avec l'axe de l'écoulement. En écoulement plan, cette surface cylindrique devient un cercle. Il est alors clair que le choc de géométrie circulaire, mais constituant un choc oblique pour l'écoulement tourbillon, voit son existence conditionnée par la valeur du nombre $M \cos \alpha$ par rapport à l'unité :

- si $\mathrm{M} \cos \alpha>1$, la recompression s'opère par choc;

- si $\mathrm{M} \cos \alpha<1$, la recompression suit un processus isentropique.

Mais, comme MM. Marchal et Servanty l'avaient exposé à la session de l'A.T.M.A. 1954 [1] la signification physique du nombre $M \cos \alpha$, mise en évidence dans le cas de l'écoulement tourbillon, présente en fait un caractère général et, dans la deuxieme partie de cette note, nous nous proposons de montrer que la relation $\mathrm{M} \cos \alpha \leqslant 1$ qui permet le retour sans onde de choc du régime supersonique au régime subsonique est tout à fait identique au critère :

$$
\frac{d \mathrm{M}^{\star}}{d \theta} \leqslant \mathrm{M}^{\star} \operatorname{tg} \lambda
$$

( $\lambda$ désignant l'angle de Mach), critère donné dans le paragraphe intitulé « Theoretical consideration of transonic flow without shocks » de l'ouvrage de A. H. Shapiro : The dynamics and thermodynamics of compressible fluid flow [2]. 


\section{Cas de l'écoulement tourbillon.}

A partir des relations :

$$
\frac{d r}{r}+\frac{d u}{u}\left(1-\mathrm{M}^{2} \cos ^{2} \alpha\right)=0
$$

el :

$$
\mathrm{R}=--\frac{u}{d u} \frac{d r}{\sin \alpha}
$$

on obtient une expression du rayon de courbure $R$ sous forme finie :

$$
\mathrm{R}=\frac{r\left(1-\mathrm{M}^{2} \cos ^{2} \alpha\right)}{\sin \alpha}
$$

comme, de plus, on a $\cos \alpha=(d r / R d \theta)$, l'ensemble de ces dernières relations permet d'écrire :

$$
\operatorname{tg} \alpha=-d \theta \frac{u}{d u}
$$

$M \cos \alpha \leqslant 1 \quad$ oll $\quad \frac{1}{\cos ^{2} \alpha} \geqslant M^{2}$

s'écrit alors :

$$
1+\left(\frac{u d \theta}{d u}\right)^{2} \geqslant \mathrm{M}^{2}
$$

soit :

$$
\frac{u d \theta}{d u} \geqslant \frac{1}{\operatorname{tg} \lambda}
$$

OU:

$$
\frac{d u}{u} \frac{1}{d \theta} \leqslant \operatorname{tg} \lambda
$$

soit enfin :

$$
\frac{d \mathrm{M}^{\star}}{\overline{\mathrm{M}}^{\star}} \frac{1}{d \theta} \leqslant \operatorname{tg} \lambda
$$

C.Q.F.D.

Les deux relations sont bien identiques l'une à l'autre.

\section{Cas général en écoulement plan.}

Reprenons la relation (3) que nous écrirons :

$$
\frac{d u}{u} \frac{\mathrm{R}}{d s} \leqslant \operatorname{tg} \lambda
$$

ds signifiant l'élément de ligne de courant.

Comme $d s=u d t$, il vient facilement :

$$
\frac{d u / d t}{u^{2} / \mathrm{R}} \leqslant \operatorname{tg} \lambda \text { (voir fig. 2) }
$$

or :

$$
\frac{d u / d t}{u^{2} / \mathrm{R}}
$$

représente la tangente de l'angle $\gamma$ que fait le vecteur accélération, c'est-à-dire la normale à l'isobare, avec la normale à la ligne de courant.

Nous en déduisons :

$$
\gamma=\frac{\pi}{2}-\alpha
$$

Nous avons done:

$$
\operatorname{tg} \gamma \leqslant \operatorname{tg} \lambda
$$

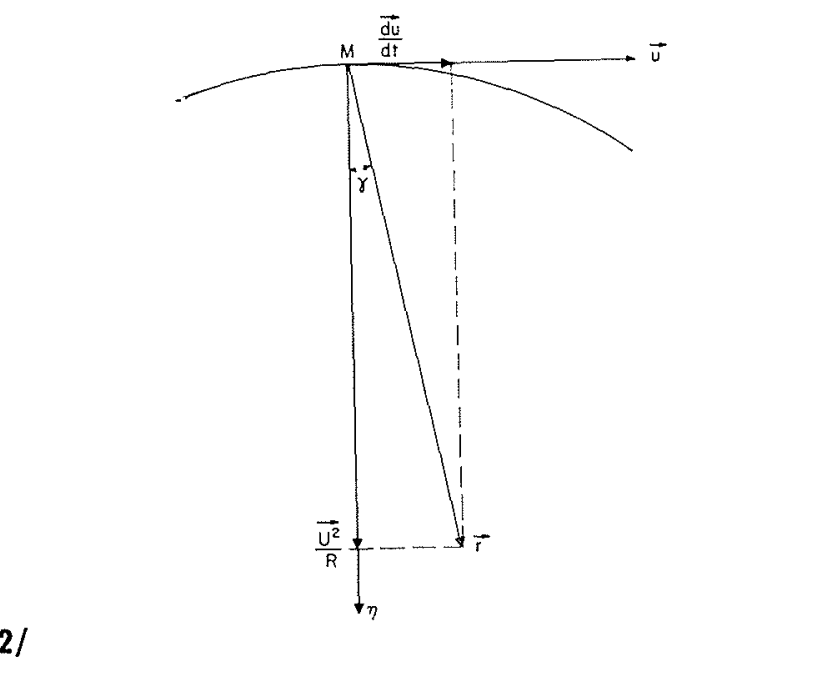

2/

soit :

$\sin \gamma \leqslant \sin \lambda$

et enfin :

$\cos \alpha \leqslant \sin \lambda$

c'est-à-dire :

$M \cos \alpha \leqslant 1$

L'identité dans le cas général des deux relations est donc parfaitement établie.

La signification physique du nombre $\mathrm{M} \cos \alpha$ qui a été noté $m$ a été donnée dans la publication [1] à laquelle nous emprunterons notre conclusion :

«En généralisant, on reconnaît que ce qui intervient, ce n'est pas la direction du rayon vecteur dans le problème de la source-tourbillon, mais bien la direction des normales aux surfaces isobares. Il faut bien admettre en effet qu'une perturbation aval ne peut modifier l'éconlement qu'à condition de modifier les conditions de pression amont, génératrices de l'écoulement. D'où l'idée que le nombre $m$ doit présenter un caractère de frontière absolument général. Quand $m$ est inférieur à 1 , les perturbations aval modifient, au cours de l'établissement du régime permanent, les surfaces de pression amont et empêchent la condensation des trains d'ondes donnant naissance aux ondes de choc. Si, au contraire, $m$ est supérieur à 1 , le maintien de l'écartement entre les surfaces isobares successives ne peut être assuré de facon directe, d'où la possibilité d'une condensation amenant progressivement la formation des ondes de choc.»

\section{Références bibliographiques}

[1] R. Marchal et P. Servaxtr. - Note sur les écoulements plans transsoniques. Ginéralisation du nombre de Mach. A.T.M.A., session 1954.

[2] A.H. Shaproo. - The dynamics and thermodynamics of compressible fluid flow, vol. II, p. 891. 


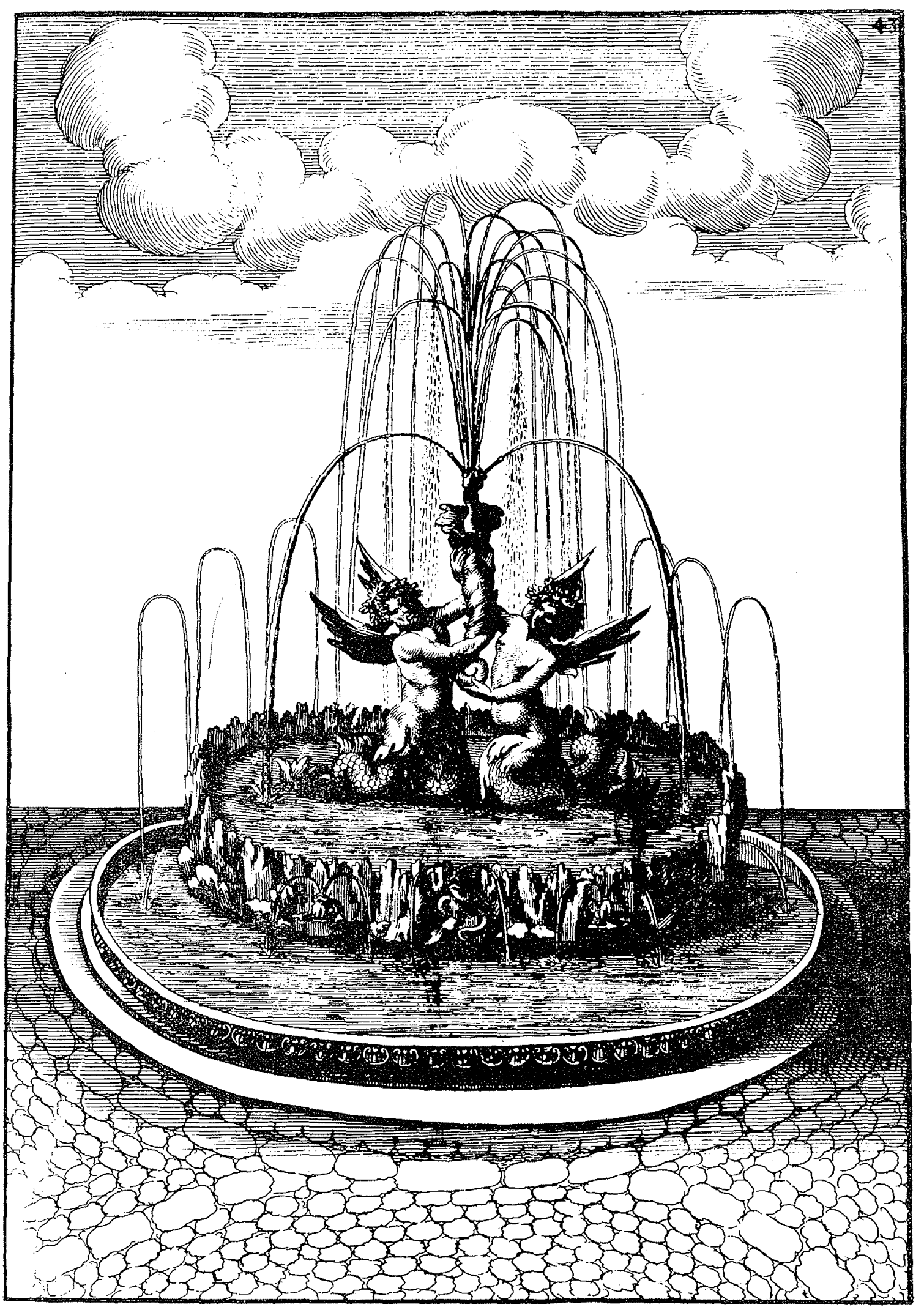

Gravure extraite de Architectura curiosa nova par G. A. BÖCKLERN Nuremberg (1664) 